\title{
Bleeding Detection in Gastrointestinal Images using Texture Classification and Local Binary Pattern Technique: A Review
}

\author{
Aparna Goyal ${ }^{1, *}$ and Reena Gunjan ${ }^{1}$ \\ ${ }^{1}$ Electronics and Communications Engineering Department, MIT School of Engineering, MIT ADT University, \\ Pune, Maharashtra, India.
}

\begin{abstract}
Texture analysis has proven to be a breakthrough in many applications of computer image analysis. It has been used for classification or segmentation of images which requires an effective description of image texture. Due to high discriminative power and simplicity of computation, the local binary pattern descriptors have been used for distinguishing different textures and in extracting texture and color in medical images. This paper discusses performance of various texture classification techniques using Contourlet Transform, Discrete Fourier Transform, Local Binary Patterns and Lacunarity analysis. The study reveals that the incorporation of efficient image segmentation, enhancement and texture classification using local binary pattern descriptor detects bleeding region in human intestines precisely.
\end{abstract}

\section{Introduction}

Bleeding in the intestine is an indication of several possible gastrointestinal (GI) diseases like ulcerative colitis (UC), tumors, and inflammatory diseases etc. Hence, detection of regions of bleeding becomes crucial in diagnosis of several diseases related to intestines. Among thousands of images received only few image frames might contain bleeding or very miniscule bleeding regions which may not be detected by manual inspection. Therefore, computerized automated methods have become the need of the hour for accurate, reliable and fast diagnosis. Among the techniques of analysis of medical images, texture analysis has played a crucial role. Texture is a tool to sub divide the images and then classify particular region of interest. It identifies the peculiarity of images and helps us in analyzing its features. Texture gives us information about structural arrangement of surfaces and their relationship to the surrounding environment. In several diseases, such as Pulmonary Fibrosis causing the thickening of tissues in the lungs, the textural changes are more visible in the X-ray images. Similar applications require more efficient texture analysis method helps to provide correct diagnosis. In gastroenterology, a distinct and specific texture pattern can be seen in ulcerous region in the internal mucous membrane of the digestive tract. The quality of the extracted features plays a crucial role in deciding the accuracy of bleeding detection method. In different medical image analysis systems, grey-scale images are used to calculate textural features. The Local Binary Pattern (LBP) operator is one of popular texture methods that has proven to perform well in texture classification applications.

\section{Related work}

The very first method of calculating LBP was introduced by Ojala[1] in 1996 which analyzed the images by their LBP histograms. Features based on histogram can identify the bleeding areas irrespective of their dimension and shape. Local Binary Pattern (LBP) is a comprehensible and an adequate texture operator. It remains uninterrupted if in gray scale occurs and provides good performance. Local Binary Pattern (LBP) is logical and consistent texture operator. It assigns labels to the pixels of an image. While labeling the pixels, its thresholds the pixels in the proximity of central pixel and assigns it a binary digit zero or one. The histogram of different intensity labels thus obtained were used for description of the texture. LBP Patterns distribution in an LBP histogram was used for analysis of texture image. Mekha Mathew, Varun P Gopi [2] had proposed a new method to discriminate bleeding regions and normal regions

\footnotetext{
*Corresponding author: aparnagoyal30@gmail.com
} 
using Local Binary Pattern (LBP) and Contourlet transform. The images were resized to $256 \times 256$. In order to focus on intrinsic geometric structure, the two-dimensional contourlet transform was applied. The contourlet transform filters the noise and detects the smooth contour in different directions of images. This transform performs 2 level decomposition resulting in coarse sub band having images of low frequency and fine sub-bands having images with high frequency. RIULBP (Rotation Invariant Local Binary Pattern) were applied on these bands. Using the combination of Laplacian Pyramid (LP) and the Directional Filter Banks (DFB) a contourlet filter bank is obtained which is a double filter bank to analyses various texture feature.

Baopu Liand Max Q.-H. Meng[3] worked on the method that emphasized upon color texture feature using Chrominance moment by dividing the image into components as luminance and chrominance. The method used Tchebichef polynomials and illumination invariance of hue/ saturation/ intensity color space. The authors have used additionally uniform local binary pattern, to differentiate bleeding and nonbleeding regions in CE images.

Another method was developed by Konstantin Pogorelov and Shipra Suman [4] to detect bleeding in GI images using color feature and texture feature. The authors have first utilized color information to detect image frames with bleeding. To modify bleeding and non-bleeding images, detection algorithm makes use of classification methods based on machine-learning to execute segmentation at pixel-level for bleeding frames in WCE images. Adriana Florentina, Mihaela [5] proposed a method for reducing the overall frames number in medical imaging so to reduce shorter analysis time. The authors had used a software application for texture analysis based on local binary pattern (LBP) operator. This operator detects and removes non-informative frames and then identifies potential lesions. Using LBP operator, the texture of intestinal mucosa, food residues and air bubbles were differentiated, then the number of non-informative frames were detected with different LBP operators. The classification of feature vectors was done using K-Means clustering technique. Yuhui Quan [6] proposed a method based upon lacunarity analysis to distinguish spatial patterns of image structure. In this work, a robust statistical approach for texture description was developed, to analyze photometric changes and geometric changes. The method incorporates multi-scale local binary patterns of image. Aliahmadvand et.al. [7] had introduced LBP with discrete wavelet transform using multi resolution analysis. By utilizing frequency information i.e., the spectral features of textures in LBP based methods, the DWT(a spatial frequency filter) was used for extraction of the spatial frequency channels and then LBP features were extracted from these channels. The combined approach using LBP and DWT improved the classification rate of LBP for invariant texture classification.

\section{Texture analysis}

The analysis of the intensities of nearby pixels in an image without consideration of their color is distinguished by the texture feature. The orientation and shape of a surface and inspection of a surface are machine visions tasks which necessitate efficient analysis of texture. It defines the intrinsic properties of surfaces especially those that do not have a smoothly varying intensity. Several image properties such as smoothness, coarseness, depth, regularity etc. can be associated with texture. Texture can also be defined as a descriptor of local brightness variation from pixel to pixel in a small neighborhood through an image. In any digital image, the spatial arrangement of the pixel defines the texture. It is a neighborhood property of an image point. The dimensions of the observation neighborhood vary according to texture measures.

Different Texture Evaluation methods depends upon the technique used for extracting features of the texture in an image. The extraction of textural features is categorized as follows: Statistical methods, Structural methods and Transform-based methods as illustrated in fig.1.

As texture is a property of spatial arrangement thus the relationship between the gray level pixel is analyzed by the statistical analysis approach using histogram. These histograms illustrate statistical moments such as variance, mean, skewness and kurtosis of the intensity histogram of an image to analyze various texture features. One of the most accepted method for texture analysis is based upon two-dimensional grey level co-occurrence matrix.

Structural texture analysis techniques represent texture as the constitution of perspicuous texture elements. The image texture is defined by the properties of the texture elements. The structural approach describes the image symbolically.

Texture analysis method such as Transform Based approach, alter images into a new domain. The intensity variations in the image pixel provides the spatial frequency. The transform technique used to separate out textural trait from the image shows the efficiency of this method. 


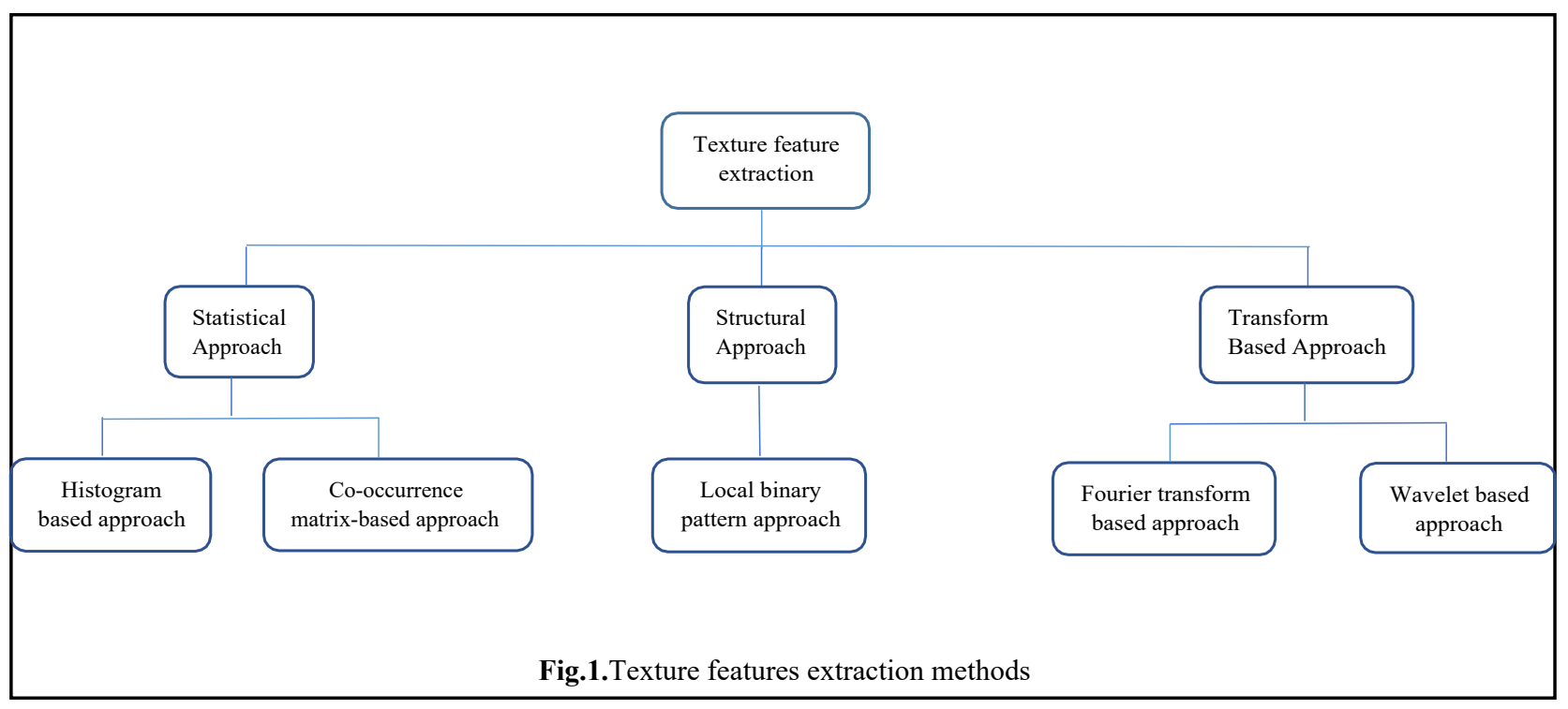

\section{Detection of bleeding in GI images}

Several methods have been developed incorporating statistical, structural and transform based approach for detection of bleeding in gastrointestinal images. A few methods have been outlined in brief using these approaches as indicated in the fig. 2 . given below.

\subsection{Contourlet Transform and Local Binary Pattern (LBP)}

By using a double filter bank structure, the contourlet transform smooths the contour of images. In double filter bank, the point discontinuities are first detected by the Laplacian pyramid (LP), then converting point discontinuities into linear structures is done by directional filter bank (DFB). The properties of contourlet transform are multi resolution, localization, directionality, critical sampling and anisotropy. Multi scale and multidimensional decomposition are its basic functions.

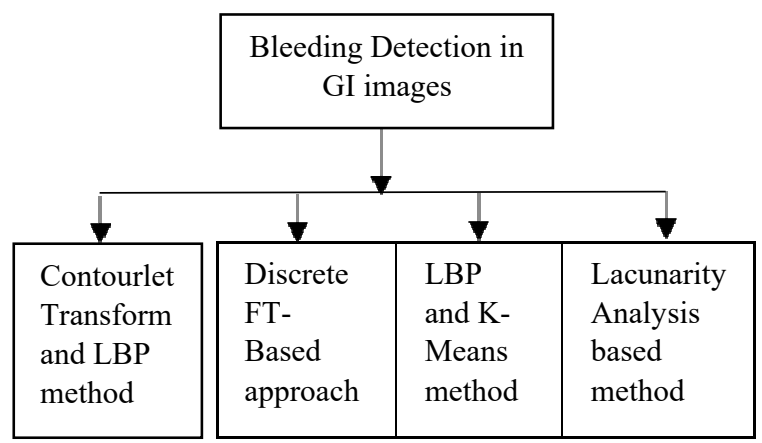

Fig. 2. Bleeding detection techniques
Local Binary Pattern (LBP), is a combination of structural and statistical approach. LBP has proven to be effective and efficient texture operator. In the image frame any monotonic transformation does not alter the corresponding LBP code and LBP is invariant against brightness

LBP divides an image into pixels. Each pixel present in a cell is compared to the neighboring pixel, which follows circular pattern that is clockwise or anticlockwise direction. If the centre pixel value is greater than the neighbor pixel, the value assigned is 0 or if not 1 is assigned.

As a result, a binary digit is obtained, which can also be named as binary string. Then histogram of the image is constructed. intensity values of pixels are shown by the histogram of an image. Finally, normalization of the histogram is done. The texture descriptor can be taken as different labels of the histogram. Thus, by the distribution of LBP patterns texture of an image can be typified by an LBP histogram.

\subsection{Discrete Fourier Transform Based Approach}

Transform methods represent an image in a space whose coordinate system defines the attribute of a texture. The application of Discrete Fourier Transform (equivalent to short time Fourier Transform) of small patches of the images is used in this feature extraction method. These images, as shown in the fig.3, are passed through frequency band filters. Inverse discrete Fourier transform is performed on resulting image and an image with frequency in selected band is obtained. Thus, rotation invariant texture descriptors are obtained in the form of local frequency descriptors. 


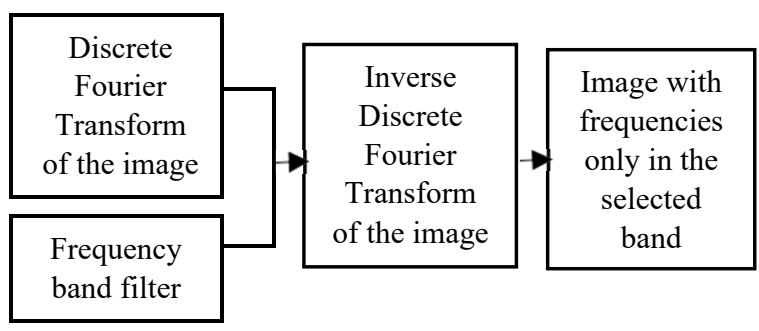

Fig. 3. Feature extraction method using DFT

\subsection{LBP and K-Means Clustering Technique}

Among various data analysis technique, clustering is used most extensively used to analyses the preliminary structure of the data set. K-Means clustering identifies subgroups in the given data set and divides the data based on the similarity in distinct cluster. This technique finds homogeneous subgroups within the data by finding Euclidean distance between the data points or correlation based distance. The determination of similarity depends upon the particular application for which K-Means clustering is used. Clustering analysis can be done based on features where subgroups of samples based on features are found. K-Means subdivides clustered observations into a specific number of disjoint clusters. This technique minimizes the distance between the centroid of the cluster and the given observation. The procedure is repeated iteratively by appending an observation to the cluster and procedure is terminated when the lowest distance measure is observed.

\subsection{Lacunarity Analysis for Texture Classification}

Lacunarity suggest how the space in the data set is filled by the pattern. Lacunarity is more if there is a wide range of gap in pattern. For homogeneous texture lacunarity should be Low and transitionally invariant, as all gap size are of the same dimension, for heterogeneous texture lacunarity is considered high. It is a scale dependent estimation of the homogeneity of the texture pattern. The method is used to discriminate the different textures incorporating local binary pattern technique. Then multi-scale local binary pattern approach is applied on images to compute lacunarity-related parameters which characterizes the texture of the image. This distinguishes the spatial patterns in the texture of the image. The spatial distribution on multiple scales characterizes the features of local image structures.

\section{Conclusion}

A large collection of accumulated images during intestinal endoscopy emphasizes the need for efficient texture-based image classification methods for better diagnosis. This review was performed to study several texture feature extraction techniques. The contourlet-based texture feature combines uniform local binary pattern approach for texture classification. The method yields good result and thus increases better diagnosis. The Fourier transform based techniques contain information confined to the frequency domain only. It cannot be used for the space domain. Since frequency spectrum does not reflect enough on the spatial description of the texture. Therefore, the frequency response thus obtained from the spectrum may not be used for the spatial localization in the image texture directly. One of the constraints of the Fourier transform being computationally complex, local variations of textures cannot be described by Fourier Transform. Henceforth, other methods such as Gabor decomposition-based approaches or wavelet-based approaches are proposed for improved spatial localization in the texture. Capturing localized frequency and spatial information is possible by the wavelet transform. The wavelet transform requires less complex computation. In order to analyze the image texture at different scales, spatial resolution can be varied using wavelet transform. However, the wavelet transform is neither translation-invariant nor rotation invariant. $\mathrm{K}$ Means clustering technique or the nearest neighbor technique is simple to implement. $\mathrm{K}$ - means classifiers are helpful in analyzing a small dataset. The Local Binary Pattern descriptors have been powerful tools for feature extraction. Texture features in Wireless Capsule Endoscopy image data set in GI tract can be extracted efficiently by incorporating proper image segmentation technique together with local binary pattern descriptors. It is observed that the methods based upon structural and statistical technique like Local Binary Pattern approach increase the performance of texture analysis. It is found that the method is simple to implement and requires less computational cost.

\section{References}

1. T. Ojala, M. Pietikainen, D. Harwood, Pattern Recognition, 29, 55, (1996).

2. M. Mathew, V.P.Gopi, IEEE Sponsored 2nd Int. Conf. on Electro. and Comm. System, 13,1223, (2016).

3. B. Li., Max Q. H. Meng, Comp. in Bio. and Medicines, 141, (2008).

4. P. Konstantin, S. Shipra, J. of Applied Clinical Medical Physics, 20, 141, (2019).

5. A. Florentina, M. Ionescu, Applied Medical Informatics Original Res., 36, 31, (2015).

6. Y. Quan, Y. Xu, Y. Sun, Y. Luo, IEEE Conf. on Computer Vision and Pattern Recog., 160, (2014).

7. A. Ahmadvand, R. Ahmadvand, M. T .Hajiali, M. Mosavi, 2nd Int. Conf. on Knowledge-Based Eng. and Innovation, 152, (2015) 